\title{
Individual differences in the detection of embedded figures*
}

\author{
HOWARD S. HOCK, GREGORY P. GORDON \\ Florida A tlantic University, Boca Raton, Florida $\mathbf{3} 3432$ \\ and \\ NORMAN MARCUS \\ The Johns Hopkins University, Baltimore, Maryland 21218
}

\begin{abstract}
The individual differences in processing obtained by Hock (1973) were extended to two experiments in which the embedded figures test was recast into the form of a yes-no reaction time task. Ss emphasizing analytic processes detected the embedded figures more rapidly than Ss emphasizing structural processes, supporting the hypothesis that attention to the parts of the embedded figures was the strategy most appropriate for the task. Based on the interaction between familiarity (normal vs rotated orientation) and embeddedness (intact vs embedded figures), it was concluded in both experiments that familiarity facilitated the detection of the embedded figures for analytic Ss. For structural Ss, familiarity was similarly found to facilitate the detection of the embedded figures, but only when a template-matching strategy was possible (when the target figure and the embedded figure were physically identical).
\end{abstract}

In a recent study using the same-different comparison task, Hock (1973) obtained evidence for individual differences in the mode of processing underlying "same" responses. These differences were indicated by a significant correlation between the effects of symmetry (symmetrical vs asymmetrical patterns) and rotation (familiar vs rotated-familiar patterns) on "same" reaction time. Those Ss whose "same" responses were faster for symmetrical than for asymmetrical patterns were said to emphasize a structural mode of processing. This was based on the hypothesis that structural processes involve rules (i.e., symmetry) for organizing the detailed parts of a stimulus into a well-formed whole. Those Ss whose "same" reaction times were unaffected by symmetry were said to emphasize an analytic mode of processing. This was based on the hypothesis, proposed initially for "different" responses, that analytic processes involve decomposing the stimulus information into features, rather than structuring the stimulus information into well-organized wholes.

The initial purpose of the present investigation was to provide further experimental support for the individual differences in processing obtained by Hock. The embedded-figures task was adopted for this purpose, since an examination of previous research (Gottschaldt, 1926: Djang, 1937; Hanawalt, 1942) had suggested that an S's ability to detect embedded figures depended on whether the $S$ emphasized structural or analytic processes. Since the embedded-figures task has had important theoretical implications with respect to the influence of familiarity on perceptual processes (Koffka, 1935; Zuckerman \& Rock, 1957), the second purpose of

*This research was supported in part by a contract between the Engineering Psychology Programs, Office of Naval Research, and The Johns Hopkins University, and in part by a Seed Grant from Florida A tlantic University. Requests for reprints should be sent to Howard S. Hock, Department of Psychology, Florida Atlantic University, Boca Raton, Florida 33432.

$\dagger$ Now at Emory University, Atlanta, Georgia 30322. this investigation was to determine whether familiarity would affect performance on the embedded-figures task when individual differences in processing were taken into account. In the experiments reported in this paper, the embedded figures task was recast into the form of a yes-no reaction time task. In addition to stimuli with embedded figures, stimuli consisting of pairs of intact, unembedded figures were also presented. On the basis of Hock's (1973) findings, individual differences in processing were determined from the effect of rotating the pairs of intact figures into an unfamiliar orientation. Emphasis on structural processes was inferred for Ss with large rotation effects; emphasis on analytic processes was inferred for Ss with small rotation effects.

Gottschaldt's (1926) embedded-figures study, which presumed that perception was based on structural or organizational processes, was concerned with the relationship between familiarity and perceptual structure. He manipulated structure by embedding simple figures in highly complex forms with new and compelling organizations, and found that camouflage was most effective when: (1) the lines of the simple figures were smoothly continued into those of the more complex form, (2) the boundary function of lines was changed such that a line would define a closed surface in the more complex form that was different from that in the simple figure, and (3) asymmetrical parts of the simple figure were incorporated into symmetrical portions of the more complex form.

If familiarity with the simple figures influenced perception, Gottschaldt contended, it would affect the structure or organization of the complex form, and result in the emergence of the familiar simple figure as a perceptual unit. Gottschaidt found, however, that regardless of whether or not the Ss were instructed to search for the simple figures, familiarity did not influence the frequency with which the simple figures were reported in the Ss' descriptions of the complex 

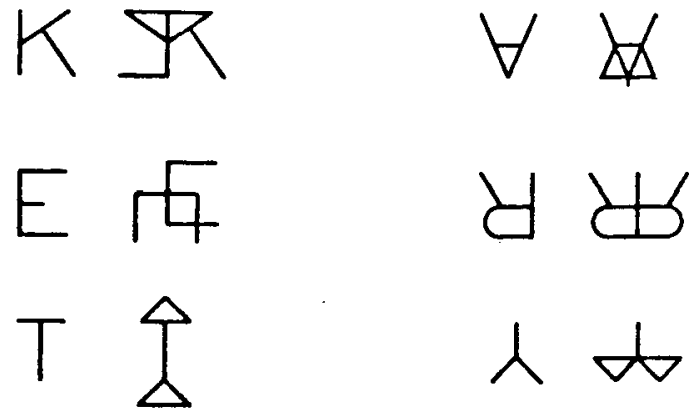

NORMAL ORIENTATION

ROTATED ORIENTATION

Fig. 1. Sample of embedded-figures stimuli.

forms. This was taken, by Gottschaldt, as evidence for the predominance of the structural properties of the complex form over the effects of familiarity.

Although Gottschaldt's study has been cited almost exclusively, a similar investigation by Djang (1937) yielded contrary results. That is, preliminary experience with simple dot patterns facilitated their identification when they were embedded in more complex dot patterns. Zuckerman and Rock (1957), in defending Gottschaldt's findings, argued that Djang's instruction to "indicate the units or sections in which you split up the figure [Djang, 1937, p. 34]" provided her Ss with an analytic set. This view was compatible with Hanawalt's (1942) finding that Ss who were successful at detecting embedded figures reported that they looked for the important parts of the figure rather than looking at the figure as a whole.

Djang's and Hanawalt's results suggest, therefore, that analytic processes may be more appropriate than structural or organizational processes for the embedded-figures task. If, as Gottschaldt demonstrated, Gestalt-type structural properties can successfully camouflage a figure, then an analytic mode of processing, sensitive to the parts of a figure rather than its overall structure, may well be the most effective way of penetrating the camouflage. It was hypothesized, therefore, that analytic Ss (those Ss with small rotation effects for the intact stimuli) would detect the embedded figures more rapidly than would structural Ss (those Ss with large rotation effects for the intact stimuli).

Regardless of which kind of process produced better performance on the embedded-figures task, however, it remained possible that familiarity would influence the detection of the embedded figures for Ss emphasızing either structural or analytic processes. On the basis of Djang's study, it was expected that familiarity would facilitate the detection of embedded figures for Ss emphasizing analytic processes. Gottschaldt's study, which presumed the operation of structural processes, suggested that familiarity would not influence the detection of embedded figures for Ss emphasizing structural processes. Any comparison between the present study and the research of Gottschaldt and Diang, however, must be qualified by the fact that the latter investigators manipulated familiarity directly (they compared familiar with unfamiliar stimuli). In the present study, familiarity was manipulated indirectly by rotating familiar stimuli into an unfamiliar orientation.

\section{EXPERIMENT I}

\section{Method}

\section{Stimuli}

The basic figures used in this experiment, as well as in the following one, were uppercase alphabet letters. Since familiarity was manipulated by presenting the letters in either their normal orientation or rotated $180 \mathrm{deg}$ into an unfamiliar orientation, letters that were invariant under $180 \mathrm{deg}$ rotation were not used. The stimuli were designed so that horizontally arranged pairs of letters were presented simultaneously, the letters being the same for half the stimuli and different for the other half. Each letter that was used appeared equally often in the left and right positions of the pair.

The intact stimuli consisted of pairs of black letters (24-pt Univers 53 Letraset) on a white background. The letters were arranged horizontally, subtending a visual angle of $1.4 \mathrm{deg}$ (at the farthest points) when the stimuli were presented in a two-channel Scientific Prototype tachistoscope.

The embedded figures stimuli consisted of a target letter (presented on the left) and a letter embedded in a complex form (presented on the right). Following Gottschaldt, the letters on the right were camouflaged by introducing symmetries, closures, and continuities into the complex forms. Although the embedded letters were not readily obvious, they could be detected more easily than was the case in Gottschaldt's study. When the embedded-figures stimuli were rotated $180 \mathrm{deg}$ into an unfamiliar orientation, both the target letter and the embedded letter were rotated, but the target letter remained in the left position. For half the stimuli, the target letter matched the embedded letter ("yes" trial); for the other half, the embedded letter was different from the target ("no" trial). Each letter was embedded in two different kinds of complex forms, with each kind appearing only twice (once in the normal orientation and once in the rotated orientation). In this way, the opportunity for the Ss to recognize the particular embeddings was minimized. A sample of the embedded figures stimuli is presented in Fig. 1.

\section{Design}

A total of 112 stimuli were assigned to eight experimental conditions, determined by the orthogonal combination of three experimental variables: yes-no, embedded-intact, and normal-rotated (there were 14 stimuli within each combination). All the stimuli were presented in a randomly mixed sequence that was different for each $S$. Before the start of the experimental trials, the Ss were shown demonstration stimuli, and were then given 16 practice trials with a mixed sequence of embedded and intact numerals.

\section{Procedure}

A small fixation dot was presented before the start of each trial. The stimulus was then presented for a period of $3 \mathrm{sec}$, unless the $S$ responded, whereupon the display was terminated. Upon the presentation of each stimulus, the Ss responded by pressing the "yes" button with a finger of their preferred hand if the target letter matched the letter on its right (for the embedded figures stimuli, the letter on the right was 
camouflaged). When there was no match, the Ss did not respond in any way. The Ss were instructed to respond as quickly as possible, within the context of keeping their errors to a minimum. The reaction time from the onset of the stimulus display was the dependent measure of primary concern

\section{Subjects}

Thirty-two male and female undergraduate students from Florida Atlantic University participated in this experiment. They were paid $\$ 1.75$ for an experimental session lasting about $25 \mathrm{~min}$.

\section{Results}

The mean reaction times for the "yes" responses of Experiment I, as well as the mean percentage errors of omission and comission, are presented in Table 1. The effects of familiarity (normal vs rotated), $F(1,31)=$ 48.44, embeddedness (intact vs embedded stimuli), $F(1,31)=248.89$, and the interaction between familiarity and embeddedness, $F(1,31)=12.92$, were all significant, $p<.005$. The overall error rate of $2.8 \%$ was divided between errors of omission and comission, with most of the errors occurring on the embedded figures stimuli (for which the error rate was 5.0\%).

The primary hypothesis for this experiment, which was concerned with individual differences in performance on the embedded-figures task, was evaluated in terms of the correlation between processing mode (structural vs analytic) and detection performance. The effect of rotation on the intact figures was used to indicate the mode of processing emphasized by each $S$ (based on Hock's 1973 findings), and the difference in reaction time between the embedded and intact stimuli (in their normal orientation) measured each S's ability to detect embedded figures. The resulting correlation, which was based on the scattergram of Fig. 2, was $\mathrm{r}=$ $0.73, \mathrm{p}<.001$. An examination of the scattergram indicated that the hypothesized results were obtained. That is, analytic Ss (as indicated by a small rotation effect for the intact figures) detected the embedded figures more rapidly than did the strustural Ss.

Table 1

Mean Reaction Times (in Milliseconds) and Mean Percentage of Errors of Omission and Comission for All Ss Combined, the 16 Ss Emphasizing Analytic Processes, and the 16 Ss Emphasizing Structural Processes (Experiment I)

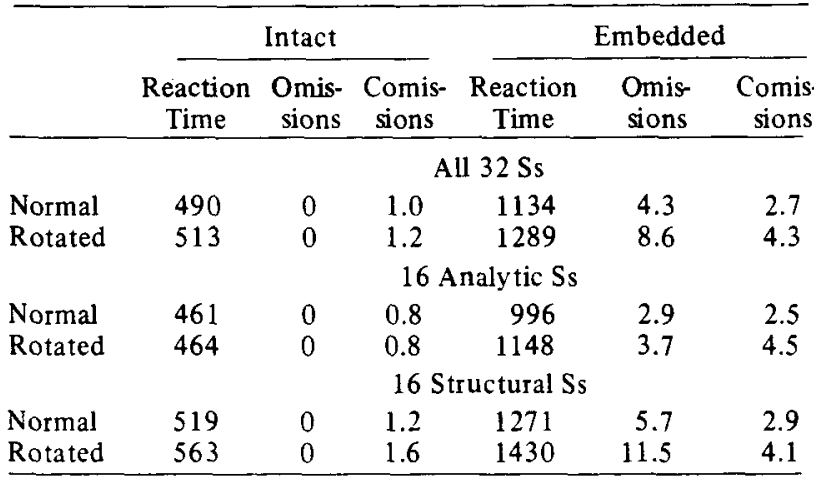

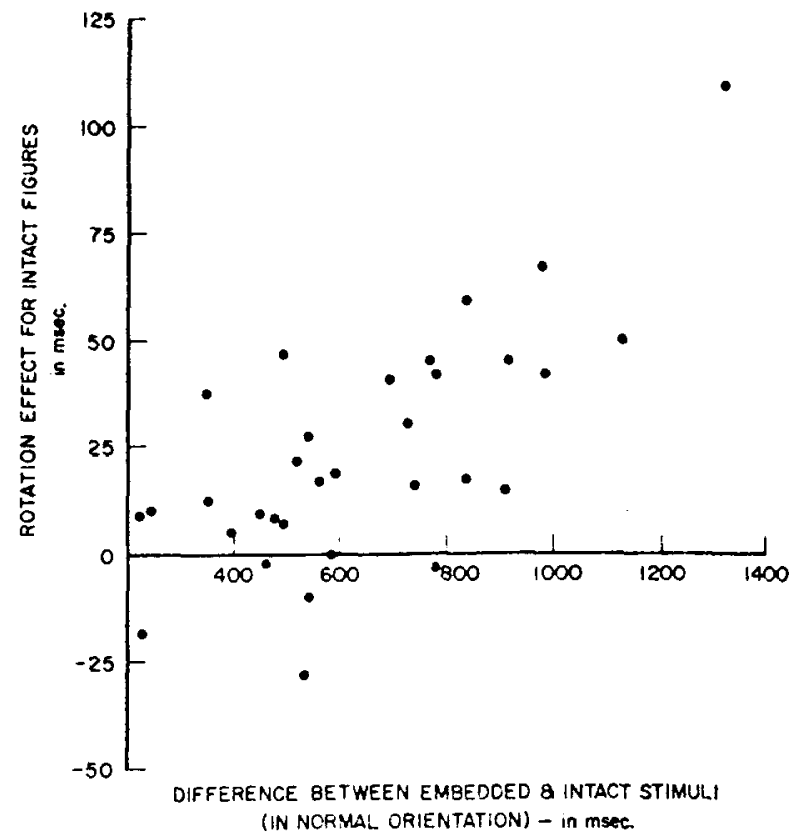

Fig. 2. Scattergram for "yes" responses of Ss in Experiment I (each point represents the data for one S).

It should be noted that the two factors entering into the above correlation were not orthogonal. Since the familiar-intact condition was included in both factors, it was possible that differences in response time for this one condition could have artifactually produced the obtained correlation. To examine this possibility, a partial correlation coefficient was computed, with performance on the familiar-intact condition "partialled out" (McNemar, 1962). The resulting correlation increased to $r=0.74$. Furthermore, since performance on the familiar-intact stimuli indicated the relative speed of each $\mathrm{S}$, the partial correlation procedure also eliminated the possibility that the obtained correlation was artifactually enhanced because slow Ss had proportionally larger rotation and embeddedness effects than fast Ss.

The second hypothesis for this experiment concerned the effect of familiarity on the detection of the embedded figures. This hypothesis was examined by subdividing the Ss into two groups: the 16 who most strongly emphasized analytic processes (the Ss with the smallest rotation effects for the intact stimuli) and the 16 who most strongly emphasized structural processes (the Ss with the largest rotation effects for the intact stimuli). As can be seen from Table 1, both the analytic and structural Ss were faster when the embedded-figures stimuli (consisting of a target letter and a letter embedded in a complex form) were presented in their familiar orientation than when they were rotated into an unfamiliar orientation. It was possible, however. that this familiarity effect may have involved the recognition of the target letters rather than the detection of the embedded letters. In order to account for the 
Table 2

Mean Reaction Times (in Milliseconds) and Mean Percentage of Errors of Omission and Comission for All Ss Combined, the 16 Ss Emphasizing Analytic Processes, and the 16 Ss Emphasizing Structural Processes

\begin{tabular}{|c|c|c|c|c|c|c|c|}
\hline & & \multicolumn{3}{|c|}{ Intact } & \multicolumn{3}{|c|}{ Embedded } \\
\hline & & $\begin{array}{c}\text { Reaction } \\
\text { Time }\end{array}$ & $\begin{array}{l}\text { Omis- } \\
\text { sions }\end{array}$ & $\begin{array}{l}\text { Comis- } \\
\text { sions }\end{array}$ & $\begin{array}{l}\text { Reaction } \\
\text { Time }\end{array}$ & $\begin{array}{l}\text { Omis- } \\
\text { sions }\end{array}$ & $\begin{array}{c}\text { Comis- } \\
\text { sions }\end{array}$ \\
\hline All 32 Ss & $\begin{array}{l}\text { Normal } \\
\text { Rotated }\end{array}$ & $\begin{array}{l}617 \\
675\end{array}$ & $\begin{array}{l}0.2 \\
0\end{array}$ & $\begin{array}{l}1.0 \\
3.1\end{array}$ & $\begin{array}{l}1295 \\
1403\end{array}$ & $\begin{array}{r}8.6 \\
12.7\end{array}$ & $\begin{array}{r}7.0 \\
10.7\end{array}$ \\
\hline 16 Analy tic Ss & $\begin{array}{l}\text { Normal } \\
\text { Rotated }\end{array}$ & $\begin{array}{l}590 \\
600\end{array}$ & $\begin{array}{l}0 \\
0\end{array}$ & $\begin{array}{l}0.8 \\
3.3\end{array}$ & $\begin{array}{l}1222 \\
1332\end{array}$ & $\begin{array}{r}9.0 \\
11.1\end{array}$ & $\begin{array}{l}6.6 \\
8.6\end{array}$ \\
\hline 16 Structural Ss & $\begin{array}{l}\text { Normal } \\
\text { Rotated }\end{array}$ & $\begin{array}{l}644 \\
750\end{array}$ & $\begin{array}{l}0.4 \\
0\end{array}$ & $\begin{array}{l}1.2 \\
2.9\end{array}$ & $\begin{array}{l}1368 \\
1474\end{array}$ & $\begin{array}{r}3.2 \\
14.4\end{array}$ & $\begin{array}{r}7.4 \\
12.7\end{array}$ \\
\hline
\end{tabular}

recognition of the target letters, the effect of familiarity on the detection of the embedded letters was assessed relative to the effect of familiarity on the intact stimuli. Interaction contrasts were computed for each $S$ by subtracting the effect of familiarity on the intact stimuli (rotated-intact minus normal-intact) from the effect of familiarity on the embedded-figures stimuli (rotated-embedded minus normal-embedded). The interaction contrast was significantly greater than zero for both the analytic Ss, $t(15)=4.12, \mathrm{p}<.001$, and the structural $\mathrm{Ss}, \mathrm{t}(15)=2.72, \mathrm{p}<.02 .{ }^{1}$ This indicated that familiarity (normal vs rotated) had facilitated the detection of embedded figures for both groups of Ss.

As in Hock's (1973) experiment, analytic Ss were faster than structural Ss. This was the case for the intact stimuli, $\mathrm{t}(30)=2.19, \mathrm{p}<.05$, as well as the intact and embedded-figures stimuli combined, $t(30)=3.86$, $\mathrm{p}<.001$. It was also found that: (1) the difference between the rotated-embedded and rotated-intact conditions was greater for the structural than for the analytic Ss, $t(30)=2.06, p<.05$, and (2) the structural Ss made more errors of omission on the embedded-figures stimuli than did the analytic Ss, $t(30)$ $=2.04, \mathrm{p} \cong .05$. Both of these findings lent further support to the correlational evidence (Fig. 2) indicating that analytic Ss perform better on the embedded-figures task than do structural Ss. Since errors of comission were similarly distributed for both groups of Ss, there was no indication that differences in reaction times for the two groups could be attributed to differences in speed-accuracy criteria.

\section{EXPERIMENT II}

Since the embedded-figures stimuli used in the preceding experiment consisted of an intact uppercase alphabet letter on the left side of the stimulus and an uppercase letter embedded in a complex form to its right, it was conceivable that at least some Ss detected the embedded letter by "mentally sliding the target letter so that it overlapped the complex form, and then shifting the position of the target until a match was obtained." In order to demonstrate that the results obtained in the previous experiment did not depend on the sort of template matching process described above, the stimuli were altered such that the target letter was always a lowercase alphabet letter. In this way, "yes" responses were based on name matches rather than the physical matches used in the previous experiments. This was the case for both the embedded-figures stimuli and the intact stimuli (intact lowercase letter on left and intact uppercase letter to its right).

In all other respects, the experimental procedure was identical to that of the preceding experiment. An additional 32 undergraduate and graduate students at Florida Atlantic Universityparticipated in this experiment, for which they were paid $\$ 1.75$.

\section{Results}

The mean reaction times for this experiment, as well as the mean percentage errors of omission and comission, are presented in Table 2. As in Experiment I, the effects of familiarity, $F(1,31)=24.11$, and embeddedness, $F(1,31)=540.10$, were both significant, $p<.005$. Although it had the same form as in Experiment $I$, the interaction between familiarity and embeddedness was insignificant, $F(1,31)=2.10, p>.05$. The overall error rate, $5.4 \%$, was due primarily to errors on the embedded-figures stimuli (for which the error rate was $9.8 \%$ ).

As in Experiment I, Ss emphasizing analytic processes detected the embedded figures more rapidly than Ss emphasizing structural processes. This was again indicated by the correlation between the effect of rotation on the intact figures (the measure of individual differences) and the difference in reaction time between the embedded and intact stimuli (in their normal orientation). The correlation, which was based on the scattergram of Fig. 3, was $r=0.40, p<.05$. The partial correlation procedure used in Experiment $I$ to account for both the absence of orthogonality and differences in performance level was repeated here. The resulting partial correlation coefficient was $r=0.37$, which was again significant, $t(29)=2.17, \mathrm{p}<.05$.

As in Experiment $I$, the 16 Ss who most strongly emphasized analytic processes were placed in one group and the 16 Ss who most strongly emphasized structural 
processes were placed in a second group (see Table 2). Once again, the effect of familiarity on the detection of embedded figures was examined on the basis of interaction contrasts (the effect of rotation on the embedded-figures stimuli minus the effect of rotation on the intact stimuli). As in Experiment I, the interaction contrast for the analytic Ss was significant, $t(15)=2.21$, $\mathrm{p}<.05$. Unlike Experiment $\mathrm{I}$, the interaction contrast was insignificantly different from zero, $t(15)>1.0$, for the structural Ss. Also unlike Experiment $I$, the difference in reaction time between the rotated-embedded and rotated-intact conditions was almost identical, $t(30)>1.0$, for the structural and analytic Ss. An examination of the errors of comission (see Table 2) suggested that these differences with respect to Experiment I may have been due to the structural Ss' trading off accuracy for speed in the rotated-embedded condition. That is, the structural Ss may have had relatively fast reaction times for the rotated-embedded stimuli because they were willing to accept a high rate of comission errors in this condition. If this were the case, however, it would be expected that the reaction times for the incorrect "yes" responses (comission errors) would be as fast, if not faster, than the reaction times for correct "yes" responses. Since the comission errors were consistently slower than the correct responses for the rotated-embedded stimuli, it could be concluded that the high error rate in this condition was not due to a speed-accuracy tradeoff.

Other than the rotated-embedded condition, the distribution of errors was similar for the structural and analytic Ss. As a final note, analytic Ss were again faster than structural Ss. This was the case for intact stimuli, $t(30)=3.33, p<.01$, as well as the intact and embedded figures stimuli combined, $\mathrm{t}(30)=2.73, \mathrm{p}<.02$.

\section{DISCUSSION}

The results of Experiments I and II supported the individual differences in processing obtained by Hock (1973). Emphasis on analytic vs structural processing was inferred from the effect of rotating the intact alphabet letters into an unfamiliar orientation, and the inference was supported by correlational evidence indicating that analytic Ss were superior at detecting the embedded figures. Individual differences in the detection of embedded figures have previously been reported by Witkin et al (1954), who found that performance in the embedded-figures task was correlated with performance on the rod-and-frame test. That is, Ss who were most successful at the detection of embedded figures were least influenced by the visual frame in aligning the rod to "vertical." Witkin's conclusion, that such "field-independent" Ss were capable of dealing analytically with a given situation, was compatible with the results obtained for the analytic Ss in the present research. Individual differences have also been obtained by Teuber and Weinstein (1956), who found that Ss

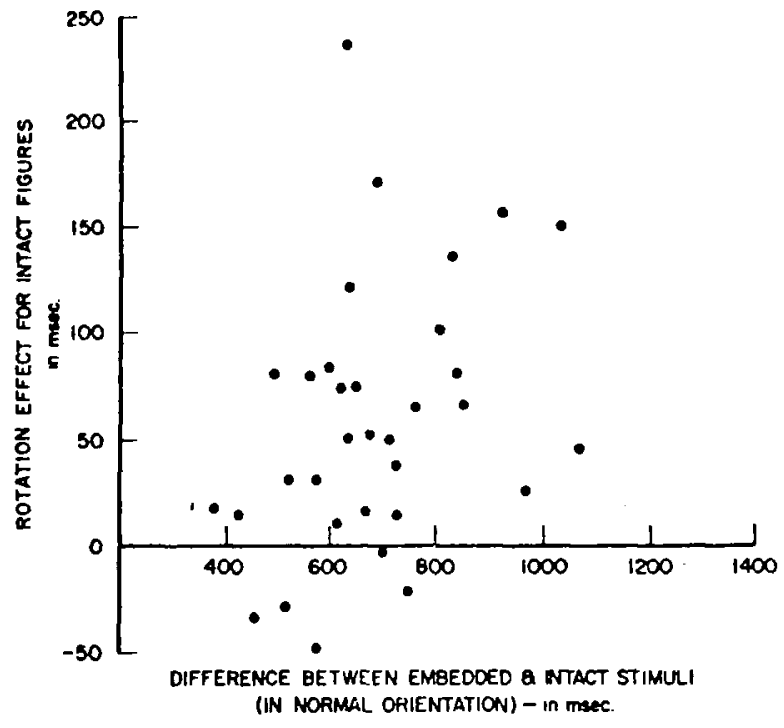

Fig. 3. Scattergram for "yes" responses of Ss in Experiment II (each point represents the data for one $S$ ).

with normal speech perform better on the embedded-figures task than do aphasics. When combined with the results of the present study, Teuber and Weinstein's study suggests that there is a close relationship between analytic and verbal processes. This possibility has been supported by evidence that verbal encoding is implicit in the analytic Ss" "same" responses to pairs of identical alphabet letters. 2

Individual differences in this study were determined from the effect of rotating familiar, intact letters into an unfamiliar orientation. Underlying this measure of individual differences was the assumption that differences in the size of $\mathrm{Ss}^{\prime}$ rotation effects reflect differences in their dependence on spatial reference. For structural Ss, the coordination of familiarity effects to a spatial frame of reference was indicated by Hock's (1973) finding that the 180-deg rotation of familiar patterns virtually eliminated the influence of familiarity on structural processes. For analytic Ss, however, Hock found that the effects of familiarity were invariant under 180-deg rotation. Whereas structural Ss organized the stimulus information with respect to a spatial reference, analytic Ss were able to process the stimulus information on the basis of features that were independent of spatial reference.

In the present study, it was possible for the analytic Ss to have differentiated between the intact alphabet letters on the basis of such rotationally invariant features as the numbers of straight lines, curved lines, right angles, and acute angles. Using these features, they could infer that a letter was an " $F$," for example, by determining that it had three straight lines and three right angles. There was no need for the analytic Ss to consider the relative location or even the orientation of the straight lines in order to identify the "F."

Such independence of spatial reference could not have 
been the case, however, when the " $F$ " was embedded in a more complex form. Not only were there more than three straight lines in the complex form, but it was necessary to consider the orientation of the lines as well as their relative spatial locations in order to detect the embedded " $F$." This need for spatial reference, involving orientation-specific relational features (e.g., the horizontal lines of the " $F$ " are to the upper right of the vertical line), would therefore explain why the analytic Ss detected the embedded letters more rapidly when they were in their familiar spatial orientation than when they were rotated into an unfamiliar orientation. In this way, when the familiar letters were degraded by embedding them in complex forms, the rotation of the embedded-figures stimuli into an unfamiliar orientation constituted a manipulation of familiarity for both structural and analytic Ss.

Whether or not familiarity influenced the detection of the embedded figures was determined by interaction contrasts that compared familiarity (normal vs rotated) effects on the embedded-figures stimuli with familiarity effects on the intact stimuli. It was found, in both Experiments I and II, that familiarity facilitated the detection of embedded figures (as indicated by the significant interaction between familiarity and embeddedness) for Ss emphasizing analytic processes. This supported Zuckerman and Rock's (1957) contention that the familiarity effect Djang (1937) obtained in her embedded-figures task was the result of the analytic set induced by her instructions. For Ss emphasizing structural processes, it was found that familiarity facilitated the detection of embedded figures in Experiment I but not in Experiment II. Although it was possible that Experiment II simply failed to completely replicate the results of Experiment I, the fact that template matching was possible in only one of the experiments suggested another interpretation of the data. In Experiment I, when it was possible for the embedded figures to be detected by a template-matching procedure, the structural Ss may have benefited from the influence of familiarity on the structural integrity of the perceived target letter. In this way, the perceptual template (target letter) could have remained more stable and unitary as the structural Ss scanned the complex form for a match, producing better performance in the familiar than in the unfamiliar orientation. When the possibility of a template match was eliminated (Experiment II), familiarity no longer influenced the structural Ss' ability to detect embedded figures (the interaction between familiarity and embeddedness was insignificant). This supported Gottschaldt's (1926) contention that familiarity with a simple figure does not influence the structural organization of the complex form in which the simple figure is embedded.

\section{REFERENCES}

Djang, $\mathrm{S}$. The role of past experience in the visual apprehension of masked forms. Journal of Experimental Psychology, 1937, 20, 29-59.

Gottschaldt, K. Gestalt factors and repetition, 1926. In W. D. Ellis (Ed.), A source book of Gestalt psychology. New York: Humanities Press, 1950.

Hanawalt, N. G. The effect of practice upon the perception of simple designs masked by more complex designs. Journal of Experimental Psychology, 1942, 31, 134-148.

Hock, H. S. The effects of stimulus structure and familiarity on same-different comparison. Perception \& Psychophysics, $1973,14,413-420$.

Koffka, K. Principles of Gestalt psychology. New York: Harcourt, Brace \& World, 1935.

McNemar, Q. Psychological statistics. New York: Wiley, 1962.

Teuber, H. L., \& Weinstein, S. Ability to discover hidden figures after cerebral lesions. Archives of Neurological Psychiatry, 1956, 76, 369-379.

Witkin, H. A., Lewis, A. B., Hertzman, M., Machover, M., Meissmer, P. G., \& Wapner, S. Personality through perception: An experimental and clinical study. New York: Harper, 1954.

Zuckerman, C. B., \& Rock, I. A reappraisal of the roles of past experience and innate organizing processes in visual perception. Psychological Bulletin, 1957, 54, 269-296.

\section{NOTES}

1. The procedure for forming the two groups of Ss biased the structural $S$ s to have a smaller interaction contrast than the analytic Ss. The purpose of this analysis, however, was to compare the interactions for each group against the null hypothesis, not to compare the size of the interaction for each group.

2. Hock and Gordon (in preparation) have found, in a same-different comparison involving physical matches (e.g., AA) and name matches (e.g., aA), that analytic Ss have smaller differences between the two types of "same" match than do structural Ss.

(Received for publication June 18, 1973; revision received August 10, 1973.) 